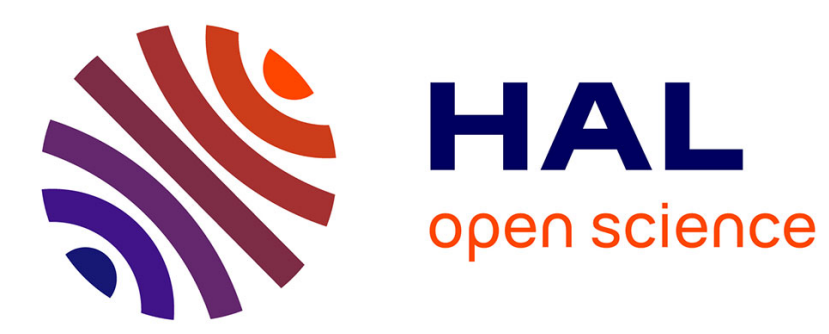

\title{
Zonotopic Kalman Filter-based Interval Estimation for Discrete-Time Linear Systems with Unknown Inputs
}

Thomas Chevet, Thach Ngoc Dinh, Julien Marzat, Zhenhua Wang, Tarek Raïssi

\section{- To cite this version:}

Thomas Chevet, Thach Ngoc Dinh, Julien Marzat, Zhenhua Wang, Tarek Raïssi. Zonotopic Kalman Filter-based Interval Estimation for Discrete-Time Linear Systems with Unknown Inputs. IEEE Control Systems Letters, 2021, 6, pp.806 - 811. 10.1109/LCSYS.2021.3086562 . hal-03239436

\section{HAL Id: hal-03239436 \\ https://hal-cnam.archives-ouvertes.fr/hal-03239436}

Submitted on 7 Jul 2021

HAL is a multi-disciplinary open access archive for the deposit and dissemination of scientific research documents, whether they are published or not. The documents may come from teaching and research institutions in France or abroad, or from public or private research centers.
L'archive ouverte pluridisciplinaire HAL, est destinée au dépôt et à la diffusion de documents scientifiques de niveau recherche, publiés ou non, émanant des établissements d'enseignement et de recherche français ou étrangers, des laboratoires publics ou privés. 


\title{
Zonotopic Kalman Filter-based Interval Estimation for Discrete-Time Linear Systems with Unknown Inputs
}

\author{
Thomas Chevet, Member, IEEE, Thach Ngoc Dinh, Julien Marzat, Zhenhua Wang, Member, IEEE, and \\ Tarek Raïssi, Senior Member, IEEE
}

\begin{abstract}
This letter proposes an unknown input zonotopic Kalman filter-based interval observer for discrete-time linear time-invariant systems. In such contexts, a change of coordinates decoupling the state and the unknown inputs is often used. Here, the dynamics are rewritten into a discretetime linear time-invariant descriptor system by augmenting the state vector with the unknown inputs. A zonotopic outer approximation of the feasible state set is then obtained with a prediction-correction strategy using the information from the system dynamics, known inputs and outputs. Bounds for both the state and unknown inputs are obtained from this zonotopic set. The efficiency of the proposed interval observer is assessed with numerical simulations.
\end{abstract}

Index Terms-Interval observer, Zonotopic Kalman filter, Unknown input, Discrete-time systems

\section{INTRODUCTION}

$\mathbf{I}$ $\mathrm{N}$ real-life applications, systems may be subject to faults and model uncertainties, which can be modeled as additive unknown inputs in the system's dynamics [1]. To be able to develop reliable control laws, it is often necessary to reconstruct these unknown signals [2]. To do so, unknown input observers have been developed [3], [4]. However, as pointed out in [5] or [6], when a system is also subject to measurement noise or disturbances, the performance of these pointwise observers may be limited. This is why set-based estimation strategies have been proposed where the noise, disturbances and initial state are assumed to be unknown but bounded. They consist in evaluating the feasible state set (FSS), i.e. the set of all states consistent with the system's dynamics and the aforementioned bounds, at each time instant and computing a lower and upper bound for each state from the knowledge of the FSS.

Directly evaluating the FSS is often a computationally complex task and set-based estimation methods then aim to find an outer approximation of this set. These methods are usually separated into two classes: interval observers [7][9] and set-membership estimation (SME) [10]-[13]. The

T. Chevet was with Conservatoire National des Arts et Métiers (CNAM), Cedric-Laetitia, 292 Rue St-Martin, 75141 Paris, France. He is now with DTIS, ONERA, Université Paris-Saclay, 91123 Palaiseau, France (e-mail: thomas.chevet@onera.fr).

T.N. Dinh and T. Raïssi are with Conservatoire National des Arts et Métiers (CNAM), Cedric-Laetitia, 292 Rue St-Martin, 75141 Paris, France (e-mail: ngoc-thach.dinh@lecnam.net, tarek.raissi@cnam.fr).

J. Marzat is with DTIS, ONERA, Université Paris-Saclay, 91123 Palaiseau, France (e-mail: julien.marzat@onera.fr).

Z. Wang is with School of Astronautics, Harbin Institute of Technology, West Da-Zhi Street, 150001 Harbin, PR China (e-mail: zhenhua.wang@hit.edu.cn). main difficulty of designing interval observers is to find a change of coordinates for the system to satisfy the so-called cooperativity property [7] since this change of coordinates greatly influences the accuracy of the observer. This difficulty can be circumvented by using SME strategies which consist in finding geometrical sets, such as ellipsoids [14], zonotopes [11], [12], [15] or polytopes [16], to approximate the FSS. While polytopic sets provide a good estimation accuracy, methods using them are computationally expensive. Zonotopic sets are a class of polytopic sets often used in set-membership strategies due to their flexibility, computational simplicity and good handling of the wrapping effect [17]. In [12], a zonotopic SME strategy that provides tighter estimates than previously introduced methods [11], [18] is proposed. This strategy constructs a family of zonotopes over-approximating the FSS and selects the best outer approximation by choosing the zonotope of minimal volume. Since this strategy obeys an update-correction strategy, it is called zonotopic Kalman filter (ZKF) [11].

Several unknown input interval observers have been proposed [5], [6], [19]. Such strategies often consider a change of coordinates aimed at separating the system into two subsystems, one of which is free from the influence of the unknown input. Classical strategies to ensure the cooperativity of the subsystem without unknown inputs are then used to obtain an interval estimate of both the state and unknown input. Such methods are bound to introduce inaccuracy in the final estimate due to the successive change of coordinates. This is why [20], [21] use a new formulation of the problem by augmenting the state vector with the unknown inputs, thus rewriting the state-space dynamics as descriptor dynamics. With usual state augmentation approaches, additional dynamics and state noise have to be considered for the extra states. The descriptor formulation then provides more degrees of freedom for the observer design by avoiding the introduction of new elements to the system's dynamics. However, the pointwise observer with propagation of the uncertainties presented in [21] and the interval observer proposed in [20] are based on the introduction of a gain matrix satisfying design constraints. On the other hand, the classical prediction-correction strategies, such as the one introduced in [12], do not require such a gain and are then more flexible. To the authors' knowledge, only a limited number of papers [20]-[23] have proposed set-membership strategies to provide guaranteed bounds simultaneously to the state and unknown inputs. Most of the works, as in [24], estimate the state by eliminating the influence of the unknown inputs. 
This letter then proposes an unknown input zonotope-based interval estimation algorithm providing guaranteed bounds for the state and unknown inputs of a discrete-time linear timeinvariant (LTI) system subject to unknown but bounded disturbances. Inspired by [12], [9] and [21], the main contributions of this study are (i) a novel zonotopic Kalman filter algorithm for discrete-time LTI systems subject to unknown input to simultaneously estimate the state and unknown inputs, allowing for more degrees of freedom and a wider application range based on the reformulation of the dynamics as a descriptor system; (ii) a new criterion to select the minimal zonotopic outer approximation of the FSS based on the minimization of the Frobenius norm of its generating matrix.

The remainder of this letter is organized as follows. Some prerequisites are introduced in Section II. Section III presents the considered formulation of the system as well as the proposed zonotopic Kalman filter algorithm. In Section IV, numerical simulation results are given to assess the efficiency of the proposed estimation strategy. Finally, Section V draws concluding remarks and perspectives.

Notations. The set of integers, real numbers and positive real numbers are denoted respectively by $\mathbb{N}, \mathbb{R}$ and $\mathbb{R}_{+}$. The set $\overline{m, n}$, with $m, n \in \mathbb{N}, m \leq n$, is the set of all $k \in \mathbb{N}$ such that $m \leq k \leq n$. The element-wise absolute value operator is denoted by $|\cdot|$. The one, Euclidean and infinity norm of a vector $x \in \mathbb{R}^{n}$ are denoted by $\|x\|_{1},\|x\|_{2}$ and $\|x\|_{\infty}$ respectively. $\mathbf{0}$ and $I$ denote respectively the zero and identity matrices of appropriate dimensions and $\mathbf{1}$ denotes the column vector of ones of appropriate dimension. The matrices $A^{\top}$ and $A^{\dagger}$, with $A \in \mathbb{R}^{n \times m}$ denote respectively the transpose and the Moore-Penrose inverse of $A$. The matrix $\operatorname{diag}(x)$, with $x \in \mathbb{R}^{n}$, is a diagonal matrix of $\mathbb{R}^{n \times n}$ having the elements of $x$ as diagonal elements. Inequalities must be understood component-wise. The Minkowski sum of two sets $A$ and $B$, i.e. the set $\{a+b \mid a \in A, b \in B\}$, is denoted by $A \oplus B$.

\section{Prerequisites and Problem Formulation}

This section introduces the necessary notions to develop the proposed set-membership estimation algorithm.

\section{A. Prerequisites on zonotopes}

A zonotope of order $r$, denoted by $\mathcal{Z}=\langle p, H\rangle \subset \mathbb{R}^{n}$, with center $p \in \mathbb{R}^{n}$ and generator matrix $H \in \mathbb{R}^{n \times r}$, is a type of polytopic set, defined as the projection by $H$ of the box $\mathbf{B}^{r}=[-1,1]^{r} \subset \mathbb{R}^{r}$ in $\mathbb{R}^{n}$ :

$$
\mathcal{Z}=\langle p, H\rangle=\left\{p+H z,\|z\|_{\infty} \leq 1\right\} .
$$

In the following, when $\mathcal{Z}=\langle p, H\rangle \subset \mathbb{R}^{n}$ is a zonotope of order $r$, it is implicitly assumed that $p \in \mathbb{R}^{n}$ and $H \in \mathbb{R}^{n \times r}$. The order is omitted if it is clear from the context or not needed for the result.

The advantage of this class of polytopic sets is that it is closed under affine transformation and Minkowski sum, i.e. if $\mathcal{Z}=\langle p, H\rangle, \mathcal{Z}_{1}=\left\langle p_{1}, H_{1}\right\rangle, \mathcal{Z}_{2}=\left\langle p_{2}, H_{2}\right\rangle \subset \mathbb{R}^{n}$ are zonotopes and $M \in \mathbb{R}^{n \times n}$, then:

$$
\begin{gathered}
M \mathcal{Z}=\langle M p, M H\rangle \\
\mathcal{Z}_{1} \oplus \mathcal{Z}_{2}=\left\langle p_{1}+p_{2},\left[\begin{array}{ll}
H_{1} & H_{2}
\end{array}\right]\right\rangle
\end{gathered}
$$

are also zonotopes. These properties make zonotopes flexible and easy to use but their complexity (i.e. their order) quickly increases when used in state estimation problems. To reduce this complexity, the reduction operator [10], [15] can be used. This operator, denoted by $\downarrow_{q}$, with $q \in \mathbb{N}$, is such that $\langle p, H\rangle \subseteq\left\langle p, \downarrow_{q} H\right\rangle \subset \mathbb{R}^{n}$. The matrix $\downarrow_{q} H$, with $H=\left[\begin{array}{lll}h_{1} & \cdots & h_{r}\end{array}\right] \in \mathbb{R}^{n \times r}$, is obtained first by sorting the $r$ columns of $H$ by decreasing order of their norm $\left\|h_{i}\right\|_{2}$, with $i \in \overline{1, r}$, and then defining:

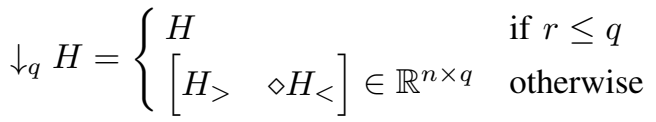

where $H_{>}$contains the first $q-n$ columns of the sorted version of $H, H_{<}$contains the last $r-q+n$ columns and $\diamond H_{<}=$ $\operatorname{diag}\left(\left|H_{<}\right| \mathbf{1}\right)[15]$.

Finally, it is necessary to introduce the notion of tight strip with respect to a zonotope and a strip [18].

Definition 1. The strip $S=\left\{x \in \mathbb{R}^{n}|| c^{\top} x-t \mid \leq \varepsilon\right\}$, with $c \in \mathbb{R}^{n}, t \in \mathbb{R}$ and $\varepsilon \in \mathbb{R}_{+}$, is called a tight strip with respect to a zonotope $\mathcal{Z} \subset \mathbb{R}^{n}$ if $\mathcal{Z} \cap S^{+} \neq \emptyset$ and $\mathcal{Z} \cap S^{-} \neq \emptyset$ where $S^{ \pm}=\left\{x \in \mathbb{R}^{n} \mid c^{\top} x=\varepsilon \pm t\right\}$.

Definition 2. Let $\mathcal{Z}=\langle p, H\rangle \subset \mathbb{R}^{n}$ be a zonotope and $F=\left\{x \in \mathbb{R}^{n}|| c^{\top} x-t_{F} \mid \leq \varepsilon_{F}\right\}$, with $c \in \mathbb{R}^{n}, t_{F} \in \mathbb{R}$ and $\varepsilon_{F} \in \mathbb{R}_{+}$, be a strip of $\mathbb{R}^{n}$ with $\mathcal{Z} \cap F \neq \emptyset$. The strip $S=\left\{x \in \mathbb{R}^{n}|| c^{\top} x-t \mid \leq \varepsilon\right\}$ where:

$$
\begin{aligned}
t & =\frac{1}{2}\left(\min \left\{q_{+}, t_{F}+\varepsilon_{F}\right\}+\max \left\{q_{-}, t_{F}-\varepsilon_{F}\right\}\right) \\
\varepsilon & =\frac{1}{2}\left(\min \left\{q_{+}, t_{F}+\varepsilon_{F}\right\}-\max \left\{q_{-}, t_{F}-\varepsilon_{F}\right\}\right)
\end{aligned}
$$

with $q_{ \pm}=c^{\top} p \pm\left\|H^{\top} c\right\|_{1}$ is called a tight strip with respect to $\mathcal{Z}$ and $F$ and is an outer approximation of $\mathcal{Z} \cap F$ such that $\mathcal{Z} \cap F=\mathcal{Z} \cap S$.

\section{B. Problem formulation}

Consider the following discrete-time LTI system:

$$
\left\{\begin{aligned}
x(k+1) & =A x(k)+B u(k)+D d(k)+D_{w} w(k) \\
y(k) & =C x(k)+D_{v} v(k)
\end{aligned}\right.
$$

where $x \in \mathbb{R}^{n_{x}}$ is the state vector, $u \in \mathbb{R}^{n_{u}}$ is the known input vector, $y \in \mathbb{R}^{n_{y}}$ is the output vector, $w \in \mathbb{R}^{n_{w}}$ and $v \in \mathbb{R}^{n_{v}}$ are respectively the disturbance and measurement noise vectors and $d \in \mathbb{R}^{n_{d}}$ is the unknown input vector. The matrices $A, B, C, D, D_{w}$ and $D_{v}$ are time-invariant matrices of appropriate dimensions.

Assumption 1. The initial state vector $x(0)$, the disturbance vector $w(k)$ and the measurement noise vector $v(k)$ are unknown but bounded and satisfy:

$$
|x(0)-p(0)| \leq x_{+} \quad|w(k)| \leq w_{+} \quad|v(k)| \leq v_{+}
$$

with $p(0) \in \mathbb{R}^{n_{x}}, x_{+} \in \mathbb{R}_{+}^{n_{x}}, w_{+} \in \mathbb{R}_{+}^{n_{w}}$ and $v_{+} \in \mathbb{R}_{+}^{n_{v}}$.

The goal of the present letter is to propose a new interval observer derived from the zonotopic set membership state estimation strategy proposed in [12] to provide guaranteed bounds to both the state vector $x$ and the unknown input vector d. 


\section{Main Result}

This section presents the system augmentation and the ZKF strategy used to obtain the intervals containing the state and the unknown input.

\section{A. System augmentation and estimation strategy}

In [5], [6], [19], [22], different strategies are proposed to decouple the unknown inputs from the state with a change of coordinates in order to find an interval containing the state vector and use this information to find an interval containing the unknown input vector. In this letter, the dynamical system (5) is rewritten into a discrete-time LTI descriptor system by considering the unknown inputs as auxiliary states [20], [21] such that:

$$
\left\{\begin{aligned}
E \bar{x}(k+1) & =\bar{A} \bar{x}(k)+\bar{B} u(k)+\bar{D}_{w} w(k) \\
y(k) & =\bar{C} \bar{x}(k)+D_{v} v(k)
\end{aligned}\right.
$$

where:

$$
\begin{aligned}
& \bar{x}(k)=\left[\begin{array}{c}
x(k) \\
d(k-1)
\end{array}\right], \quad E=\left[\begin{array}{cc}
I & -D \\
\mathbf{0} & \mathbf{0}
\end{array}\right], \quad \bar{A}=\left[\begin{array}{cc}
A & \mathbf{0} \\
\mathbf{0} & \mathbf{0}
\end{array}\right], \\
& \bar{B}=\left[\begin{array}{l}
B \\
\mathbf{0}
\end{array}\right], \quad \bar{D}_{w}=\left[\begin{array}{c}
D_{w} \\
\mathbf{0}
\end{array}\right], \quad \bar{C}=\left[\begin{array}{ll}
C & \mathbf{0}
\end{array}\right]
\end{aligned}
$$

and choosing $d(-1)=\mathbf{0}$.

Assumption 2. The matrices $C$ and $D$ satisfy the rank condition:

$$
\operatorname{rank}\left[\begin{array}{cc}
I & -D \\
C & \mathbf{0}
\end{array}\right]=n_{x}+n_{d} .
$$

The goal is then to find two bounds $\bar{x}_{u}$ and $\bar{x}_{l}$ for the augmented state vector $\bar{x}$ such that:

$$
\bar{x}_{l}(k) \leq \bar{x}(k) \leq \bar{x}_{u}(k), \forall k \in \mathbb{N} .
$$

To do so, it is necessary to introduce the following lemma.

Lemma 1 ([25]). Given matrices $X \in \mathbb{R}^{n \times m}, Y \in \mathbb{R}^{m \times p}$ and $Z \in \mathbb{R}^{n \times p}$ with $\operatorname{rank} Y=p$, the general solution $X$ of the equation $X Y=Z$ is:

$$
X=Z Y^{\dagger}+\Xi\left(I-Y Y^{\dagger}\right)
$$

where $\Xi \in \mathbb{R}^{n \times m}$ is an arbitrary matrix.

With Lemma 1 and Assumption 2, it is possible to find two matrices $T$ and $N$ such that:

$$
T E+N \bar{C}=I
$$

with:

$$
T=\Theta^{\dagger} \lambda_{1}+\Xi \Psi \lambda_{1} \quad N=\Theta^{\dagger} \lambda_{2}+\Xi \Psi \lambda_{2}
$$

where $\Xi \in \mathbb{R}^{\left(n_{x}+n_{d}\right) \times\left(n_{x}+n_{d}+n_{y}\right)}$ is an arbitrary matrix and:

$$
\Theta=\left[\begin{array}{l}
E \\
\bar{C}
\end{array}\right], \quad \Psi=I-\Theta \Theta^{\dagger}, \quad \lambda_{1}=\left[\begin{array}{l}
I \\
\mathbf{0}
\end{array}\right], \quad \lambda_{2}=\left[\begin{array}{l}
\mathbf{0} \\
I
\end{array}\right] .
$$

Using relation (10), the descriptor system (7) satisfies [20]:

$$
\left\{\begin{aligned}
\bar{x}(k+1) & =\xi(k)+N y(k+1)-N D_{v} v(k+1) \\
\xi(k) & =T \bar{A} \bar{x}(k)+T \bar{B} u(k)+T \bar{D}_{w} w(k) \\
y(k) & =\bar{C} \bar{x}(k)+D_{v} v(k)
\end{aligned}\right.
$$

In order to find the bounds of $\bar{x}(k)$ from (9), it is then necessary to characterize the FSS $\mathcal{X}(k)$ of $\bar{x}(k)$, i.e. all states $\bar{x}(k)$ compatible with the evolution model (7) and with the noise bounds and initial state set described in Assumption 1. Exact computation of the FSS is often a complex task. Therefore, an approach of lower complexity consists in computing a minimal zonotopic outer approximation of the FSS with a two-step strategy [12].

If there exists a zonotope $\overline{\mathcal{X}}(k-1)$ containing $\bar{x}(k-$ $1)$, the first step, called time update step, consists in using (12a) to obtain a new zonotope $\widetilde{\mathcal{X}}(k)$ containing $\bar{x}(k)$. Then, exploiting the fact that, by (12c), $\bar{x}(k) \in F(k)=$ $\left\{x \in \mathbb{R}^{n_{x}+n_{d}}|| \bar{C} x-y(k)|\leq| D_{v} \mid v_{+}\right\}$, it is possible to find $\mathcal{X}(k)=\langle\bar{p}(k), \bar{H}(k)\rangle$, a zonotopic minimal outer approximation of $\widetilde{\mathcal{X}}(k) \cap F(k)$. This second step is the observation update step and the set $\overline{\mathcal{X}}(k)$ is the minimal zonotopic outer approximation of $\mathcal{X}(k)$. Finally, the bounds in (9) are such that:

$$
\left\{\begin{array}{l}
\bar{x}_{l}(k)=\bar{p}(k)-|\bar{H}(k)| \mathbf{1} \\
\bar{x}_{u}(k)=\bar{p}(k)+|\bar{H}(k)| \mathbf{1}
\end{array}\right.
$$

The following paragraphs detail the two steps of the estimation strategy.

\section{B. Time update}

The time update step of the ZKF is based on the following theorem.

Theorem 1. Consider the descriptor system (7) satisfying Assumption 2 and three zonotopes $\overline{\mathcal{X}}(k)=\langle\bar{p}(k), \bar{H}(k)\rangle \subset$ $\mathbb{R}^{n_{x}+n_{d}}, \mathcal{W}=\langle\mathbf{0}, W\rangle \subset \mathbb{R}^{n_{w}}$ and $\mathcal{V}=\langle\mathbf{0}, V\rangle \subset \mathbb{R}^{n_{v}}$. If at time $k, w(k) \in \mathcal{W}, v(k) \in \mathcal{V}$ and $\bar{x}(k) \in \overline{\mathcal{X}}(k)$, then $\bar{x}(k+1) \in \widetilde{\mathcal{X}}(k+1)=\langle\widetilde{p}(k+1), \widetilde{H}(k+1)\rangle$ where:

$$
\begin{aligned}
\widetilde{p}(k+1) & =T \bar{A} \bar{p}(k)+T \bar{B} u(k)+N y(k+1) \\
\widetilde{H}(k+1) & =\left[\begin{array}{lll}
T \bar{A} \downarrow_{q} \bar{H}(k) & T \bar{D}_{w} W & -N D_{v} V
\end{array}\right]
\end{aligned}
$$

with $q \in \mathbb{N}$.

Proof. From (12a) and (12b):

$$
\begin{aligned}
\bar{x}(k+1) \in T \bar{A} \overline{\mathcal{X}}(k) \oplus T \bar{B}\langle u(k), \mathbf{0}\rangle \oplus T \bar{D}_{w} \mathcal{W} \\
\oplus N\langle y(k+1), \mathbf{0}\rangle \oplus\left(-N D_{v} \mathcal{V}\right) .
\end{aligned}
$$

However, by definition of the reduction operator $\downarrow_{q}$, with $q \in$ $\mathbb{N}, \overline{\mathcal{X}}(k) \subseteq\left\langle\bar{p}(k), \downarrow_{q} \bar{H}(k)\right\rangle$, thus:

$$
\begin{aligned}
\bar{x}(k+1) & \in T \bar{A}\left\langle\bar{p}(k), \downarrow_{q} \bar{H}(k)\right\rangle \oplus T \bar{B}\langle u(k), \mathbf{0}\rangle \\
& \oplus T \bar{D}_{w} \mathcal{W} \oplus N\langle y(k+1), \mathbf{0}\rangle \oplus\left(-N D_{v} \mathcal{V}\right)
\end{aligned}
$$

or $\bar{x}(k+1) \in\langle\widetilde{p}(k+1), \widetilde{H}(k+1)\rangle$, with $\widetilde{p}(k+1)$ and $\widetilde{H}(k+1)$ as in (14), using properties (2) and (3) on (15).

From Assumption 1 and the definition of the descriptor vector $\bar{x}$, it is possible to define a zonotope $\overline{\mathcal{X}}(0)=$ $\langle\bar{p}(0), \bar{H}(0)\rangle \subset \mathbb{R}^{n_{x}+n_{d}}$, with $\bar{p}^{\top}(0)=\left[\begin{array}{ll}p^{\top}(0) & \mathbf{0}\end{array}\right]$ and $\bar{H}(0)=\operatorname{diag}\left(\left[\begin{array}{ll}x_{+}^{\top} & \mathbf{0}\end{array}\right]\right)$, such that $\bar{x}(0) \in \overline{\mathcal{X}}(0)$. With the definition of this zonotope, and considering $\mathcal{W}=\langle\mathbf{0}, W\rangle \subset$ $\mathbb{R}^{n_{w}}$ and $\mathcal{V}=\langle\mathbf{0}, V\rangle \subset \mathbb{R}^{n_{v}}$, with $W=\operatorname{diag}\left(w_{+}\right)$and $V=\operatorname{diag}\left(v_{+}\right)$, the assumptions of Theorem 1 are satisfied 
at time $k=0$. Then, even without the second step of the $\mathrm{ZKF}$, it is possible to find a zonotope containing $\bar{x}(k)$ at all time $k>0$ by taking $\overline{\mathcal{X}}(k)=\widetilde{\mathcal{X}}(k)$ and applying Theorem 1 with the zonotopes $\mathcal{W}$ and $\mathcal{V}$ previously defined.

The reduction operator is used in Theorem 1 to limit the increasing complexity of the zonotopes involved in the estimation process. The reduction order influences both the complexity of the algorithm and the accuracy of the final interval.

\section{Observation update}

The observation update uses the information from (12c) to tighten the zonotopic set $\widetilde{\mathcal{X}}(k)$ containing $\bar{x}(k)$ obtained from Theorem 1 into a zonotopic set $\overline{\mathcal{X}}(k)$ of minimal size.

From (12c), at time $k$, the descriptor vector $\bar{x}(k)$ belongs to the set:

$$
F(k)=\bigcap_{i=1}^{n_{y}} F_{i}(k)
$$

where the $F_{i}$, with $i \in \overline{1, n_{y}}$, are strips of $\mathbb{R}^{n_{x}+n_{d}}$ :

$$
F_{i}(k)=\left\{x \in \mathbb{R}^{n_{x}+n_{d}}|| \bar{C}_{i} x-y_{i}(k) \mid \leq\left(\left|D_{v}\right| v_{+}\right)_{i}\right\}
$$

with $\bar{C}_{i}, y_{i}(k)$ and $\left(\left|D_{v}\right| v_{+}\right)_{i}$ the $i$-th row of $\bar{C}, y(k)$ and $\left|D_{v}\right| v_{+}$, respectively. Given that $\bar{x}(k) \in \widetilde{\mathcal{X}}(k) \cap F(k)$, this intersection is not empty and is tighter than $\widetilde{\mathcal{X}}(k)$. The set $\overline{\mathcal{X}}(k)$ is thus obtained by computing an outer approximation of $\widetilde{\mathcal{X}}(k) \cap F(k)$. However, computing $\overline{\mathcal{X}}(k)$ from $\widetilde{\mathcal{X}}(k) \cap F(k)$ would lead to a conservative result [12].

In the following, it is considered that $n_{y}=1$, so that $F(k)=F_{1}(k)$. The case $n_{y}>1$ is treated at the end of the present section.

To obtain a less conservative outer approximation of $\widetilde{\mathcal{X}}(k) \cap F_{1}(k)$, it is necessary to introduce the set $S_{1}(k)=$ $\left\{x \in \mathbb{R}^{n_{x}+n_{d}}|| \bar{C}_{1} x-t_{1} \mid \leq \varepsilon_{1}\right\}$, with $t_{1} \in \mathbb{R}$ and $\varepsilon_{1} \in \mathbb{R}_{+}$ obtained with Definition 2, which is not empty and is a tight strip with respect to $\widetilde{\mathcal{X}}(k)$ and $F_{1}(k)$. Since, by Definition 2 , $\widetilde{\mathcal{X}}(k) \cap F_{1}(k)=\widetilde{\mathcal{X}}(k) \cap S_{1}(k), \overline{\mathcal{X}}(k)$ is obtained by computing an outer approximation of $\widetilde{\mathcal{X}}(k) \cap S_{1}(k)$.

To this end, [12] proposes the following theorems to obtain a family of zonotopes $\mathcal{T}_{j}(k)=\left\langle\tau_{j}(k), T_{j}(k)\right\rangle \subset \mathbb{R}^{n_{x}+n_{d}}$ of order $r$, with $j \in \overline{0, r}$ and $r$ the order of $\widetilde{\mathcal{X}}(k)$, such that $\widetilde{\mathcal{X}}(k) \cap S_{1}(k) \subseteq \mathcal{T}_{j}(k), \forall j \in \overline{0, r}$. Before introducing these theorems, it is noted that, since $\bar{x}(k) \in \widetilde{\mathcal{X}}(k)=\langle\widetilde{p}(k), \widetilde{H}(k)\rangle$, $\exists z \in \mathbf{B}^{r}=[-1,1]^{r}$ such that $\bar{x}(k)=\widetilde{p}(k)+\widetilde{H}(k) z$. Moreover, given that $\bar{x}(k) \in S_{1}(k)$ :

$$
z \in G_{1}(k)=\left\{z \in \mathbb{R}^{r}|| \bar{C}_{1} \widetilde{H}(k) z-\left(t_{1}-\bar{C}_{1} \widetilde{p}(k)\right) \mid \leq \varepsilon_{1}\right\}
$$

which is a tight strip with respect to $\mathbf{B}^{r}$ [12].

Lemma 2 ([12]). Let $S=\left\{x \in \mathbb{R}^{n}|| c^{\top} x-t \mid \leq \varepsilon\right\}$, with $c \in \mathbb{R}^{n}, t \in \mathbb{R}$ and $\varepsilon \in \mathbb{R}_{+}$, be a tight strip with respect to a zonotope $\langle p, H\rangle \subset \mathbb{R}^{n}$ of order $r$. Then, the minimal volume box containing $G \cap \mathbf{B}^{r}$, where $G=$ $\left\{z \in \mathbb{R}^{r}|| c^{\top} H z-\left(t-c^{\top} p\right) \mid \leq \varepsilon\right\}$, is the zonotope $\langle b, L\rangle \subset$ $\mathbb{R}^{r}$ of order $r$, with $L$ diagonal such that:

$$
b_{i}=\frac{1}{2}\left(\alpha_{i}^{+}-\alpha_{i}^{-}\right) \operatorname{sgn}\left(c^{\top} h_{i}\right), \quad L_{i}=\frac{1}{2}\left(\alpha_{i}^{+}+\alpha_{i}^{-}\right)
$$

where $b_{i}, h_{i}$ and $L_{i}$ are respectively the $i$-th element of $b$, column of $H$ and diagonal element of $L$, with $i \in \overline{1, r}$, sgn is the sign function, and:

$$
\alpha_{i}^{ \pm}= \begin{cases}\min \left\{\frac{\varepsilon \pm\left(t-c^{\top} p\right)+\left|c^{\top} H\right| \mathbf{1}}{\left|c^{\top} h_{i}\right|}-1,1\right\} & \text { if } c^{\top} h_{i} \neq 0 \\ 1 & \text { otherwise }\end{cases}
$$

Theorem 2 ([12]). Let $S=\left\{x \in \mathbb{R}^{n}|| c^{\top} x-t \mid \leq \varepsilon\right\}$, with $c \in \mathbb{R}^{n}, t \in \mathbb{R}$ and $\varepsilon \in \mathbb{R}_{+}$, be a tight strip with respect to a zonotope $\mathcal{Z}=\langle p, H\rangle \subset \mathbb{R}^{n}$ of order $r$. Let $\langle b, L\rangle \subset \mathbb{R}^{r}$ be the box obtained from $S$ and $\mathcal{Z}$ with Lemma 2. Then, $\mathcal{Z} \cap S \subseteq$ $\mathcal{T}_{j}=\left\langle\tau_{j}, T_{j}\right\rangle \subset \mathbb{R}^{n}, \forall j \in \overline{0, r}$, where:

$$
\begin{aligned}
\tau_{j} & = \begin{cases}p_{b}+\frac{t-c^{\top} p_{b}}{c^{\top} h_{j}} h_{j} & \text { if } j \in \overline{1, r} \text { and } c^{\top} h_{j} \neq 0 \\
p_{b} & \text { otherwise }\end{cases} \\
T_{j} & =\left\{\begin{array}{lll}
{\left[\begin{array}{lll}
t_{1, j} & \cdots & t_{r, j}
\end{array}\right]} & \text { if } j \in \overline{1, r} \\
H L & \text { otherwise }
\end{array} c^{\top} h_{j} \neq 0\right.
\end{aligned}
$$

where $h_{i}$, with $i \in \overline{1, r}$, is the $i$-th column of $H, p_{b}=p+H b$ and:

$$
t_{i, j}= \begin{cases}L_{i}\left(h_{i}-\frac{c^{\top} h_{i}}{c^{\top} h_{j}} h_{j}\right) & \text { if } i \neq j \\ \frac{\varepsilon}{c^{\top} h_{j}} h_{j} & \text { if } i=j\end{cases}
$$

$\forall i \in \overline{1, r}$, with $L_{i}$ the $i$-th diagonal element of $L$.

The zonotopes $\mathcal{T}_{j}(k)=\left\langle\tau_{j}(k), T_{j}(k)\right\rangle \subset \mathbb{R}^{n_{x}+n_{d}}$, with $j \in \overline{0, r}$ and $r$ the order of $\mathcal{X}(k)$, obtained from Theorem 2 , are outer approximations of $\widetilde{\mathcal{X}}(k) \cap S_{1}(k)$ and thus of the FSS. By definition, $\overline{\mathcal{X}}(k)$ is the zonotope $\mathcal{T}_{j}(k)$ of minimal size. In this letter, the size of a zonotope is understood as the sum of squares of its generators (also known as the Frobenius norm of the generator matrix). Therefore, computing $j^{*}$ as:

$$
j^{*}=\arg \min _{j \in \overline{0, r}} \operatorname{tr}\left(T_{j}(k) T_{j}^{\top}(k)\right),
$$

$\mathcal{T}_{j *}(k)$ is the set $\overline{\mathcal{X}}(k)$, minimal zonotopic outer approximation of the FSS.

The new criterion (18) is a contribution with respect to [12], which considers the volume of the zonotopes $\mathcal{T}_{j}(k)$, as defined in [18], as the minimization criterion. On the one hand, the computation time of the volume of a zonotope is higher than the computation time of the Frobenius norm. On the other hand, the Frobenius norm is more conservative than the volume in evaluating the size of a zonotope. Therefore, the proposed minimization criterion is a good trade-off between computation burden and conservatism.

When the system has more than one output, i.e. when $n_{y}>$ 1, Algorithm 1 is used. This algorithm guarantees that the zonotope $\overline{\mathcal{X}}(k)=\langle\bar{p}(k), \bar{H}(k)\rangle \subset \mathbb{R}^{n_{x}+n_{d}}$ obtained at time $k$ is a minimal outer approximation of the FSS since, according to (16) and Definition 2:

$$
\begin{aligned}
\tilde{\mathcal{X}}(k) \cap F(k)=\tilde{\mathcal{X}}(k) \cap \bigcap_{i=1}^{n_{y}} F_{i}(k) & \subseteq \widetilde{\mathcal{X}}_{1}(k) \cap \bigcap_{i=2}^{n_{y}} F_{i}(k) \\
& \subseteq \widetilde{\mathcal{X}}_{n_{y}}(k)=\overline{\mathcal{X}}(k)
\end{aligned}
$$

where the $\widetilde{\mathcal{X}}_{i}(k)$, with $i \in \overline{0, n_{y}}$, are defined in Algorithm 1 .

Finally, the bounds $\bar{x}_{u}(k)$ and $\bar{x}_{l}(k)$ satisfying (9) are computed with (13). 


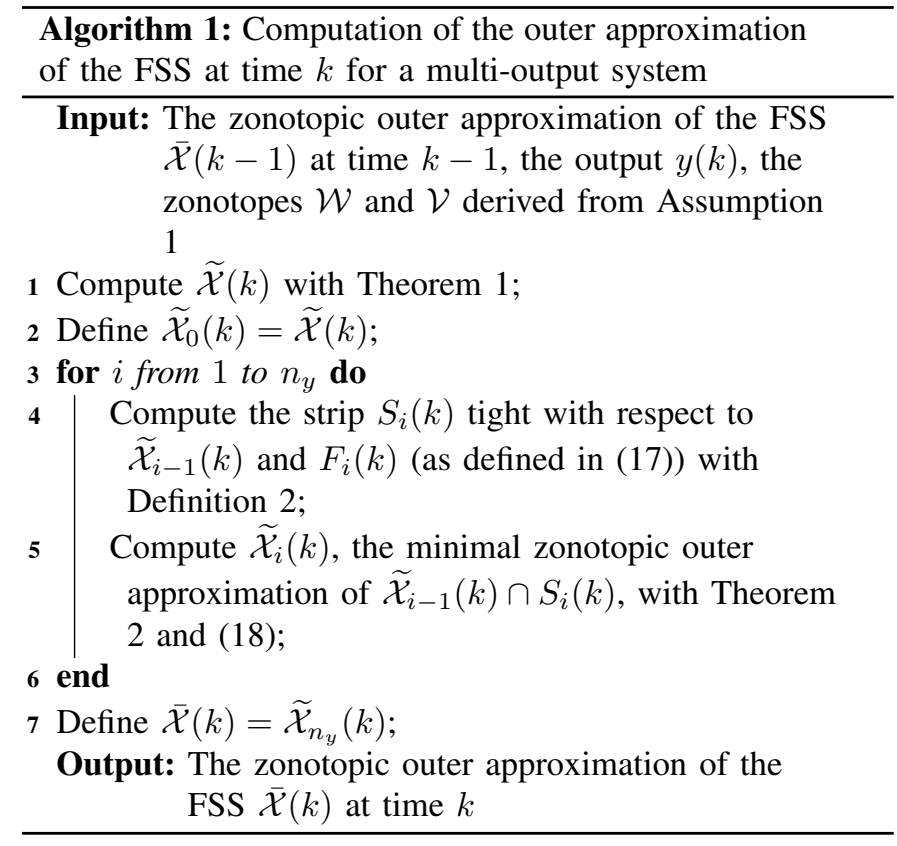

\section{Numerical Simulation}

Consider the discrete-time LTI system (5) where:

$$
\begin{array}{lcc}
A=\left[\begin{array}{ccc}
0.2 & 0.4 & 0.1 \\
0 & 0.7 & 0.2 \\
0 & 0 & 0.5
\end{array}\right] & B=\left[\begin{array}{l}
0.3 \\
0.8 \\
0.1
\end{array}\right] \quad D=\left[\begin{array}{c}
0.5 \\
1 \\
0.5
\end{array}\right] \\
C=\left[\begin{array}{ccc}
0.3 & 0.1 & 0 \\
0 & 0.2 & 0.1
\end{array}\right] & d_{w}=\left[\begin{array}{l}
0.1 \\
0.8 \\
0.3
\end{array}\right] & d_{v}=\left[\begin{array}{l}
0.5 \\
0.4
\end{array}\right]
\end{array}
$$

and $D_{w}=\operatorname{diag}\left(d_{w}\right)$ and $D_{v}=\operatorname{diag}\left(d_{v}\right)$. The disturbance and measurement noise vector are randomly chosen and satisfy respectively $|w(k)| \leq w_{+}$and $|v(k)| \leq v_{+}, \forall k \in \mathbb{N}$, where $w_{+}=0.06 \cdot \mathbf{1}$ and $v_{+}=0.06 \cdot \mathbf{1}$. The input signal is $u(k)=$ $\sin (0.02 \pi k)$. The unknown input is $d(k)=0.3 \sin (0.05 k)$. The values of $p(0)$ and $x_{+}$defined in Assumption 1 are $p(0)=$ $\mathbf{0}$ and $x_{+}=0.1 \cdot \mathbf{1}$. The reduction order for the time update step is $q=20$.

The value of $\Xi$ in (11) can be chosen as the one minimizing the estimation error $\bar{x}(k)-\widetilde{p}(k)$ as in [26] or the volume of $\widetilde{\mathcal{X}}(k)$ as in [24]. However, for the sake of simplicity, it is considered in this example that $\Xi=\mathbf{0}$. The matrices $T$ and $N$ satisfying (10) are:

$$
\begin{gathered}
T=\left[\begin{array}{cccc}
0.6645 & -0.2882 & -0.0882 & 0 \\
-0.5716 & 0.3905 & -0.2095 & 0 \\
-0.2787 & -0.3071 & 0.8929 & 0 \\
-0.5858 & -0.6047 & -0.2047 & 0
\end{array}\right] \\
N=\left[\begin{array}{cc}
1.1185 & 0.8815 \\
1.9052 & 2.0948 \\
0.9289 & 1.0711 \\
1.9526 & 2.0474
\end{array}\right] .
\end{gathered}
$$

To assess the efficiency of the method proposed in this letter, it is compared with the interval observer proposed in [6], where the observer gain is obtained by a Schur stable pole assignment $\{0.1,0.5\}$, and with the zonotopic observer
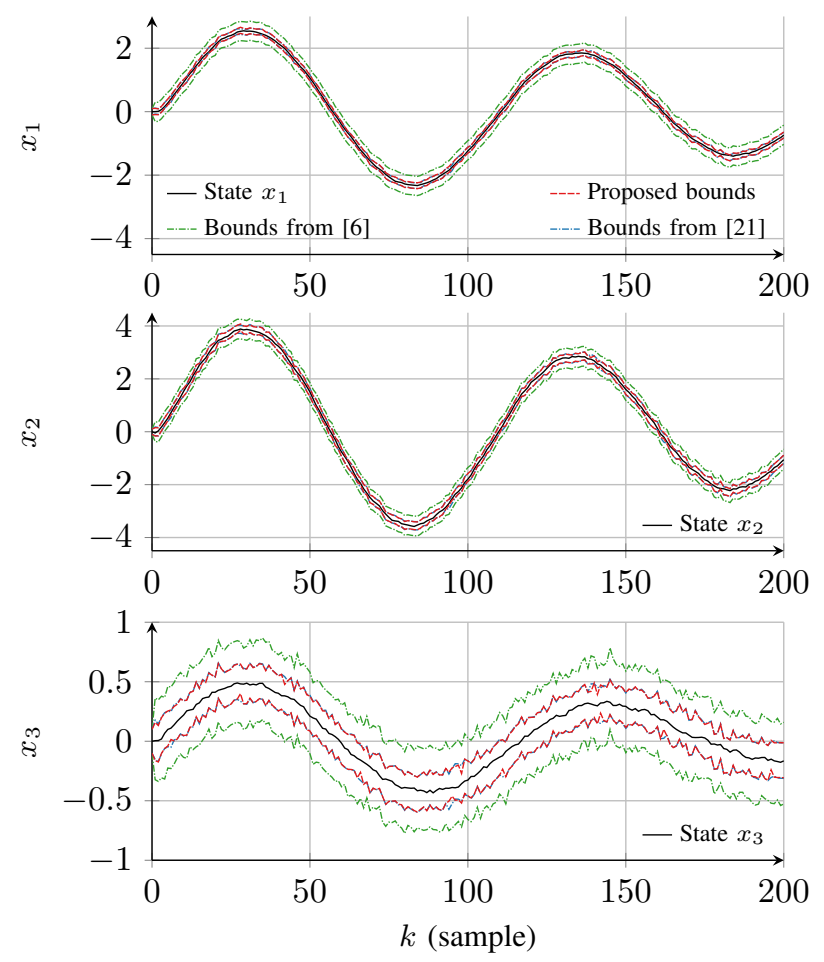

Fig. 1. States and guaranteed bounds from the proposed observer and the observers from [6] and [21]

proposed in [21]. Fig. 1 presents, in solid lines, the simulated value of the three components of the state vector $x(k)$ over time, in dashed red lines, the lower and upper bounds for each of these components obtained with the zonotope-based strategy presented in this letter, in dash-dotted green lines, the bounds obtained with the method from [6] and, in dash-dotted blue line, the bounds obtained with the observer from [21]. Fig. 2 presents the unknown input $d(k)$ in solid line, the lower and upper bounds obtained with the method presented in this letter in dashed red lines, the bounds obtained with the method from [6] with dash-dotted green lines and the bounds obtained with the observer from [21] with dash-dotted blue lines. From these figures, it can be seen that the the proposed zonotope-based interval observer performs as expected since (9) is satisfied for the three states as well as for the unknown input. Moreover, it gives bounds tighter than the observer based on successive changes of coordinates proposed in [6] and on par with the bounds obtained from the pointwise observer with propagation of the uncertainties presented in [21], as can be seen in Fig. 1 and 2.

Another way to evaluate the accuracy of the proposed observer is to compare the average estimation bounds, i.e. the average value of the difference between the upper bound $\bar{x}_{u}$ and the lower bound $\bar{x}_{l}$, i.e. the average value of $\bar{x}_{u}(k)-\bar{x}_{l}(k)$, for both observers. The average values for the three states and the unknown input for both methods are gathered in Table I. This table then confirms the fact that the proposed observer provides bounds tighter than the observer from [6] on this example. It also confirms that the performance of the proposed observer is on par with the observer from [21] on this example. 


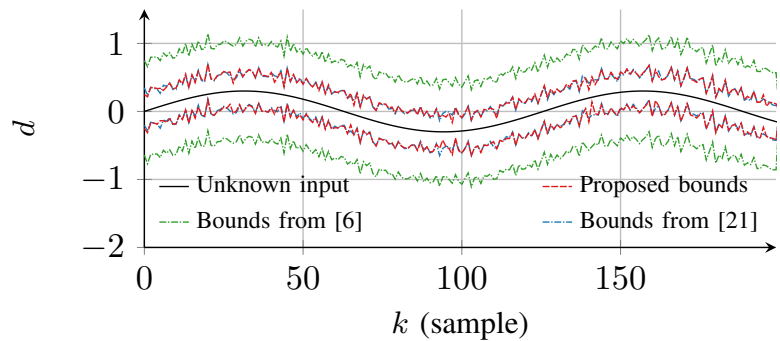

Fig. 2. Unknown input and guaranteed bounds from the proposed observer and the observers from [6] and [21]

TABLE I

AVERAGE ESTIMATION BOUNDS FOR THE STATES AND UNKNOWN INPUT

\begin{tabular}{lllll}
\hline Observer & $x_{1}$ & $x_{2}$ & $x_{3}$ & $d$ \\
\hline Observer from [6] & 0.6021 & 0.7428 & 0.6831 & 1.4273 \\
Observer from [21] & 0.1773 & 0.3097 & 0.2971 & 0.5103 \\
Proposed observer & 0.1856 & 0.2924 & 0.2894 & 0.5119 \\
\hline
\end{tabular}

In the present case, the value of the matrices $T$ and $N$ has been chosen arbitrarily. Therefore, by tuning $T$ and $N$ adequately, the performance of the proposed observer might be improved to provide tighter bounds than [21].

\section{CONCLUSION AND Future WORK}

A new zonotopic Kalman filter-based interval observer for a discrete-time linear time-invariant system subject to unknown inputs and unknown but bounded perturbation and measurement noise is studied. The initial system is rewritten into a discrete-time linear time-invariant descriptor system by augmenting the state vector with the unknown input vector. As with classical Kalman filter, the interval estimation process is divided into two steps at each time instant. The first step uses the information from the system's dynamics to derive a zonotope containing the augmented state at a given time instant from the knowledge of a zonotope containing the augmented state at the previous time instant. The second step uses the information on the output of the system to reduce the size of the previously found zonotope. The interval observer is then able to simultaneously provide bounds to both the state vector and the unknown input vector. To assess its efficiency, the proposed observer was compared in simulation with two other state-unknown inputs interval observers existing in the literature based respectively on a change of coordinates and on a pointwise observer with propagation of the uncertainties. In future work, a formal proof of convergence should be formulated. In addition this method could be extended to linear parameter-varying state space or descriptor systems subject to unknown inputs.

\section{REFERENCES}

[1] A. Varga, Solving fault diagnosis problems. Springer, 2017.

[2] P. Frank and X. Ding, "Survey of robust residual generation and evaluation methods in observer-based fault detection systems," J. Process Control, vol. 7, no. 6, pp. 403-424, 1997.

[3] M. Hou and P. Müller, "Design of observers for linear systems with unknown inputs," IEEE Trans. Autom. Contr., vol. 37, no. 6, pp. 871875, 1992.
[4] D. Maquin, B. Gaddouna, and J. Ragot, "Estimation of unknown inputs in linear systems," in Proc. Am. Control Conf., 1994, pp. 1195-1197.

[5] L. Meyer, D. Ichalal, and V. Vigneron, "Interval observer for LPV systems with unknown inputs," IET Control Theory Appl., vol. 12, no. 5 , pp. 649-660, 2018.

[6] E. I. Robinson, J. Marzat, and T. Raïssi, "Prognosis of uncertain linear time-invariant discrete systems using unknown input interval observer," Int. J. Control, vol. 93, no. 11, pp. 2690-2706, 2020.

[7] F. Mazenc and O. Bernard, "Interval observers for linear time-invariant systems with disturbances," Automatica, vol. 47, no. 1, pp. 140-147, 2011.

[8] T. Raïssi, D. Efimov, and A. Zolghadri, "Interval state estimation for a class of nonlinear systems," IEEE Trans. Autom. Contr., vol. 57, no. 1, pp. 260-265, 2012.

[9] Z. Wang, C.-C. Lim, and Y. Shen, "Interval observer design for uncertain discrete-time linear systems," Syst. Control Lett., vol. 116, pp. 41-46, 2018.

[10] C. Combastel, "A state bounding observer based on zonotopes," in Proc. IEEE ECC, 2003, pp. 2589-2594.

[11] T. Alamo, J. M. Bravo, and E. F. Camacho, "Guaranteed state estimation by zonotopes," Automatica, vol. 41, no. 6, pp. 1035-1043, 2005.

[12] W. Chai, X. Sun, and J. Qiao, "Set membership state estimation with improved zonotopic description of feasible solution set," Int. J. Robust Nonlinear Control, vol. 23, no. 14, pp. 1642-1654, 2013.

[13] W. Tang, Z. Wang, Q. Zhang, and Y. Shen, "Set-membership estimation for linear time-varying descriptor systems," Automatica, vol. 115, no. 108867,2020

[14] D. Merhy, C. Stoica Maniu, T. Alamo, E. F. Camacho, S. Ben Chabane, T. Chevet, M. Makarov, and I. Hinostroza, "Guaranteed set-membership state estimation of an octorotor's position for radar applications," Int. J. Control, vol. 93, no. 11, pp. 2760-2770, 2020.

[15] C. Combastel, "A state bounding observer for uncertain non-linear continuous-time systems based on zonotopes," in Proc. 44th IEEE Conf. Decis. Control, 2005, pp. 7228-7234.

[16] S. V. Raković and D. Q. Mayne, "State estimation for piecewise affine, discrete time systems with bounded disturbances," in Proc. 43rd IEEE Conf. Decis. Control, 2004, pp. 3557-3562.

[17] W. Kühn, "Rigorously computed orbits of dynamical systems without the wrapping effect," Computing, vol. 61, pp. 47-67, 1998.

[18] J. M. Bravo, T. Alamo, and E. F. Camacho, "Bounded error identification of systems with time-varying parameters," IEEE Trans. Autom. Contr., vol. 51, no. 7, pp. 1144-1150, 2006.

[19] D. Gucik-Derigny, T. Raïssi, and A. Zolghadri, "A note on interval observer design for unknown input estimation," Int. J. Control, vol. 89, no. 1, pp. 25-37, 2016.

[20] J. Li, T. Raïssi, Z. Wang, X. Wang, and Y. Shen, "Interval estimation of state and unknown input for linear discrete-time systems," J. Franklin Inst., vol. 357, no. 11, pp. 9045-9062, 2020.

[21] W. Zhang, Z. Wang, T. Raïssi, Y. Wang, and Y. Shen, "A state augmentation approach to interval fault estimation for descriptor systems," Eur. J. Control, vol. 51, pp. 19-29, 2020.

[22] P. Guerra, V. Puig, and M. Witczak, "Robust fault detection with unknown-input interval observers using zonotopes," IFAC Proc. Volumes, vol. 41, no. 2, pp. 5557-5562, 2008.

[23] F. Xu, J. Tan, X. Wang, V. Puig, H. Liu, B. Liang, and B. Yuan, "Robust state estimation and fault detection combining unknown input observer and set-membership approach," in Proc. SysTol'16, 2016, pp. 67-72.

[24] J. Li, Z. Wang, T. Raïssi, and Y. Shen, "Unknown input observer design for linear parameter-varying systems in a bounded error context," IEEE Trans. Autom. Contr., 2021.

[25] C. R. Rao and S. K. Mitra, "Generalized inverse of a matrix and its applications," in Proc. 6th Berkeley Symp. on Math. Statist. and Prob., vol. 1,1972 , pp. 601-620.

[26] W. Zhang, Z. Wang, T. Raïssi, T. N. Dinh, and G. M. Dimirovski, "Zonotope-based interval estimation for discrete-time linear switched systems," IFAC-PapersOnLine, vol. 52, no. 2, pp. 4707-4712, 2020. 